\title{
Recent Progress in Perturbative Quantum Field Theory*
}

\author{
Zvi Bern ${ }^{a \dagger}$

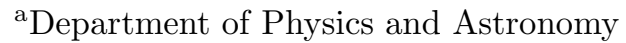 \\ University of California at Los Angeles \\ Los Angeles, CA 90095
}

In this talk, the recent breakthrough in two-loop perturbative calculations is reviewed, with emphasis on the applications to phenomenological studies. The recent precision measurement of the anomalous magnetic moment of the muon by the Brookhaven $g-2$ collaboration is also used as an illustrative example of the importance of Standard Model precision perturbative calculations to further our understanding of Nature.

\section{INTRODUCTION}

Precision calculations in the Standard Model have a long history dating back to Schwinger's original QED calculations. The importance of precision Standard Model calculations in modern day particle physics was recognized by the 1999 Nobel Prize awarded to Gerard 't Hooft and Martinus Veltman. The tools and theoretical foundation provided by 't Hooft and Veltman have led to a plethora of precision calculations, including the famous 'blueband' plot, shown many times at this conference, bounding the mass of a Standard Model Higgs boson, $m_{H} \lesssim 196-230 \mathrm{GeV}$ at $95 \%$ CL [1].

In this talk we describe recent progress in precision calculations, as well as present examples illustrating the importance of higher loop computations for comparing theory to experiments. The most spectacular recent example of this is the precision measurement of the anomalous magnetic moment of the muon by the Brookhaven $g-2$ collaboration [2,3, 3, 5]. This experiment is an ideal example due to its impressive sensitivity to the quantum corrections arising from all three components of the Standard Model: Quantum Electrodynamics, Electroweak Theory, and Quantum Chromodynamics. The incredible agreement of

\footnotetext{
*Plenary talk at XXXI International Conference on High Energy Physics (ICHEP), Amsterdam, July 2002.

${ }^{\dagger}$ This work was supported by the US Department of Energy under grant DE-FG03-91ER40662.
}

approximately seven digits of accuracy between theory and experiment is a testament to the remarkable experimental and theoretical efforts that have gone into this attempt to discover new physics beyond the Standard Model.

This talk also focuses on the very recent breakthrough in our ability to calculate two-loop quantum corrections when more than a single kinematic invariant is present. This breakthrough is the culmination of many years of theoretical effort. To illustrate the promise of this breakthrough, we present two very recent applications improving our understanding of Higgs physics, which is of central importance in particle physics. As one example, just two weeks before the conference, Anastasiou and Melnikov [6] computed the exact NNLO result for next-to-next-to-leading order (NNLO) QCD corrections to inclusive Higgs production, in perfect agreement with the earlier series expansion result of Harlander and Kilgore [7. The second example, completed a month prior to the conference, is gluon fusion into a photon pair 8.9]. This is a significant component of the background to the preferred search mode for discovering and measuring the Higgs mass at the Large Hadron Collider, if the Higgs is light $\left(M_{H}<140 \mathrm{GeV}\right)$, as suggested by precision electroweak measurements.

These examples represent initial steps in applying the breakthrough to phenomenology. It is now clear that over the next few years, NNLO calculations of jet observables at both hadron and 
lepton colliders will become available 10, 11, 12 . (Other related issues may be found in Frixione's 13 QCD theory talk.) Precision calculations of Bhabha scattering is another example that will surely benefit from the breakthrough 14, 15]. This process is important for measuring luminosity at $e^{+} e^{-}$colliders. More generally, many observables which could not previously be calculated to the required order of perturbation theory necessary to match experimental precisions will now be computed.

This talk is organized as follows. In Section 2 the theoretical calculations required for a precision prediction of the anomalous magnetic moment of the muon are reviewed briefly. In Section 3 some examples illustrating the clever tricks that enter challenging perturbative calculations are given. A brief overview of the status of loop calculations is presented in Section 4 In Section 5 we discuss the recent breakthrough in our ability to compute quantities of interest with more than a single kinematic variable. In Section 6 two very recent examples of applications of the new advances to phenomenology are presented. Prospects for the future are described in Section 7 .

\section{EXAMPLE: ANOMALOUS MAG- NETIC MOMENT CALCULATIONS}

The anomalous magnetic moment of the muon is an especially interesting quantity because of its sensitivity to high scale new physics. It is about $40,000 \simeq\left(m_{\mu} / m_{e}\right)^{2}$ times more sensitive than the electron magnetic moment in most potential scenarios of physics beyond the Standard Model. The incredible precision of both the theoretical predictions and the experiments, coupled with its sensitivity to potential new physics makes measurement of the anomalous magnetic moment of the muon an important means of searching for new physics beyond the Standard Model (see e.g. refs. [16.17]).

The magnetic moment of an elementary particle is related to it spin via the gyromagnetic ratio:

$\vec{\mu}=g \frac{e}{2 m} \vec{S}$,

where $\vec{\mu}$ is the magnetic moment and $\vec{S}$ the spin.

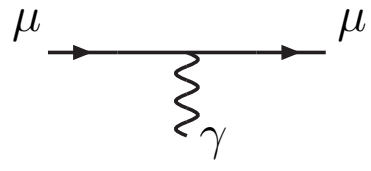

Figure 1. The Feynman diagram describing the Dirac prediction for the magnetic moment of the muon.

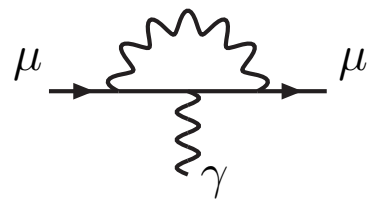

Figure 2. The Feynman diagram containing the leading quantum correction to the magnetic moment of a lepton, first obtained by Schwinger.

From the Dirac equation, the gyromagnetic ratio is $g=2$ for spin $1 / 2$ particles such as the muon or the electron. The tree-level Feynman diagram describing the Dirac prediction is depicted in Figure 1. Quantum mechanical effects represented by loop diagrams such as the one in Figure 2 , however, alter this result very slightly leading to a non-vanishing 'anomalous' magnetic moment:

$a_{\mu}=\frac{g-2}{2}$.

Computations of anomalous magnetic moments have a long history dating back to Schwinger's original calculation of the $\mathcal{O}(\alpha)$ contribution in 1948 [18].

The incredibly precise experimental result announced at this conference by Yannis Semertzidis [- 4 on behalf of the Brookhaven muon $g-2$ collaboration [3] is

$a_{\mu}^{\exp }=11659204(7)(5) \times 10^{-10}$,

which is a significant improvement over the previous measurement [2,

$a_{\mu}^{\exp }=11659202(15) \times 10^{-10}$,

and an even greater improvement on the series of experiments at CERN that ended in 1977. 
A discrepancy between the experimental result and the theoretical prediction using the Standard Model can in principle indicate new physics beyond the Standard Model,

$a_{\mu}^{\exp }-a_{\mu}^{\mathrm{SM}}=a_{\mu}^{\text {new physics }}$.

In Figure 3 a sample diagram which could lead to a New Physics contribution to the anomalous magnetic moment is shown. The virtual $\tilde{\nu}$ particle depicted in the diagram would be some as yet undiscovered particle coming from a New Physics scenario, such as supersymmetry.

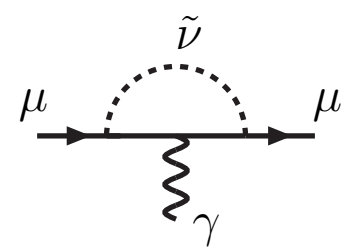

Figure 3. New physics can lead to a contribution to the anomalous magnetic moment beyond that found in the Standard Model. The dotted line labeled by $\tilde{\nu}$ represents a particle of a New Physics scenario.

The initial Brookhaven [2] result caused some excitement in the community [17 because of a $2.6 \sigma$ discrepancy between theory and experiment. However, as described below in the subsection on hadronic contributions, a correction was made in the theoretical computation that significantly reduces the discrepancy. Amusingly, the improved experimental results announced at this conference bring back the discrepancy to essentially the original level. However, the theoretical uncertainties associated with the hadronic corrections are still the subject of intense debate and it is not yet clear if the discrepancy is due to new physics.

For the purposes of this talk what is important is not the small discrepancy between theory and experiment, but the astonishing agreement to better than six significant digits. This agreement is a great triumph for modern particle physics, from both the experimental and theoretical sides.

On the theoretical side the computation requires a firm grasp of all components of the Stan- dard Model. We now briefly review the intense theoretical effort that has gone into producing Standard Model predictions with the requisite precision. More thorough reviews may be found elsewhere [16, 19,5$]$.

\subsection{QED contributions}

Calculations of the QED contributions to the anomalous magnetic moment of the muon and other leptons have a long history (see e.g. refs. 20,21,22 ) starting with the seminal work of Schwinger [18]. The QED effects are by far the largest of the contributions, but are under the best theoretical control.
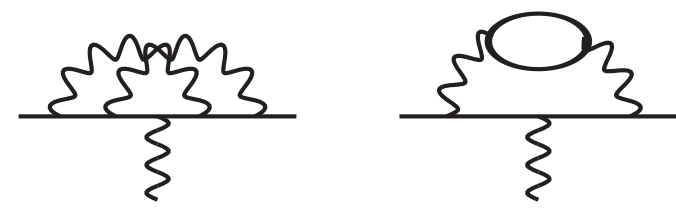

Figure 4. Examples of two-loop QED contributions to the anomalous magnetic moment.

The combined results of many years of effort is that the QED contribution is

$$
\begin{aligned}
a_{\mu}^{\mathrm{QED}}= & \frac{\alpha}{2 \pi}+0.765857376(27)\left(\frac{\alpha}{\pi}\right)^{2} \\
& +24.05050898(44)\left(\frac{\alpha}{\pi}\right)^{3} \\
& +126.07(41)\left(\frac{\alpha}{\pi}\right)^{4} \\
& +930(170)\left(\frac{\alpha}{\pi}\right)^{5}+\cdots
\end{aligned}
$$

These results are from the theory update of Czarnecki and Marciano 16], compiling the results of refs. [23,22]. The four-loop $(\alpha / \pi)^{4}$ contributions, for example, consist of a total of 891 Feynman diagrams which were evaluated by Kinoshita and his collaborators [21] via numerical means. (A recent correction actually shifts this very slightly, but with negligible effect on the prediction of the muon anomalous magnetic moment 24.) The five loop $(\alpha / \pi)^{5}$ corrections are not exact calculations but are instead estimated using various approximations [25]. An important contribution 
which significantly reduces the uncertainty is the exact analytical result for the three-loop computation [22]. The net result for the QED contribution to the anomalous magnetic moment of the muon is:

$a_{\mu}^{\mathrm{QED}}=11658470.59(29) \times 10^{-10}$.

Although the QED contribution is the largest of the contributions, the theoretical uncertainty is tiny compared to the experimental uncertainty in eq. (3) due to the high order of these calculations.

\subsection{Electroweak contributions}

The electroweak contribution is much smaller than the QED contribution because of the suppression due to $W$ or $Z$ propagators. Nevertheless, the Brookhaven experiment is sensitive to these tiny corrections. The one-loop electroweak corrections displayed in Figure 0 were computed about 30 years ago [26]. The more recently computed two-loop corrections [27 are surprisingly large, shifting the one-loop results by about $23 \%$. This large shift is due mainly to the appearance of large logarithms of the form $\ln \left(M_{Z}^{2} / M_{\mu}^{2}\right)$. The two-loop electroweak contribution is non-trivial to calculate since it is described by a total of 1650 Feynman diagram of which about 200 are important. Two sample diagrams are depicted in Figure 6. The final result for the electroweak contributions including the two loop corrections is 27

$a_{\mu}^{\mathrm{EW}}=15.1(4) \times 10^{-10}$.

The theoretical uncertainty in this quantity is tiny compared to the uncertainty in the experimental measurement (3). However, the overall contribution of the expression is well within the sensitivity of the experiment, providing an exquisitely indirect confirmation of electroweak theory [3. 4 .

\subsection{Hadronic contributions}

The most problematic of the contributions are the hadronic corrections to the anomalous magnetic moment. The underlying difficulty is that Quantum Chromodynamics is strongly interacting, making it much more difficult to carry out computations. At the low energies relevant for

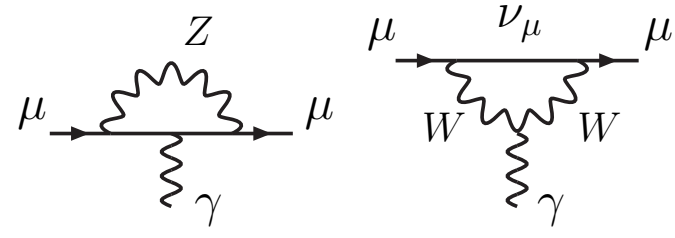

Figure 5. One-loop electroweak Feynman diagrams contributing to the anomalous magnetic moment of the muon.

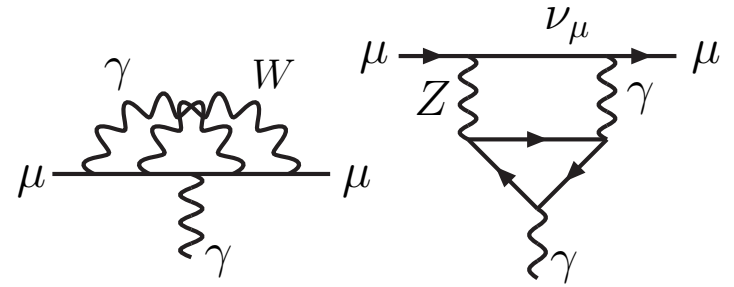

Figure 6. Two of the 1650 two-loop electroweak Feynman diagrams contributing to the anomalous magnetic moment of the muon.

the muon anomalous magnetic moment, the QCD coupling becomes large and perturbation theory breaks down. As yet, there are no available methods for performing a first principles computation of the hadronic contributions, though perhaps in the future lattice gauge theory may be able to provide some useful input. Currently, experimental input and model calculations are used.

The two basic categories of hadronic contributions are the vacuum polarization and light-bylight contributions depicted in Figure 7 and 8 . The more significant vacuum polarization contributions shown in Figure 7 can be obtained from measurements of the annihilation cross section $e^{+} e^{-} \rightarrow$ hadrons, by making use of the optical theorem and dispersion relations (see, for example, refs. [28]). The light-by-light contributions depicted in Figure 8, however, can only be obtained via model calculations with relatively large theoretical uncertainties 29]. However, since the latter contributions are much smaller, the vacuum polarization uncertainty dominates. The overall value of the light-by-light contributions, has recently been dramatically modified because of the discovery of a sign error in previous calculations [30]; this correction helps reduce the dis- 
crepancy between theory and experiment.

The status of the uncertainties in the hadronic corrections was reviewed at this conference by Teubner [5]. Since the time of the conference a number of new theoretical analyses have also appeared [31. Depending on various assumptions, currently the discrepancy is somewhere between 1.6 and $3 \sigma$. It will, however, be some time before there is a final consensus on its significance.

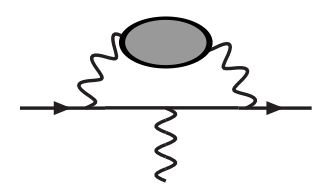

Figure 7. The hadronic vacuum polarization contribution. The wavy lines are photons and the blob represents hadronic contributions.

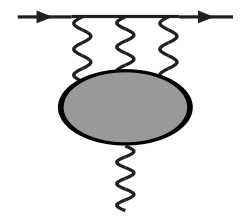

Figure 8. The hadronic light-by-light contribution.

\section{CHALLENGING PERTURBATIVE COMPUTATIONS}

The area of higher order perturbative computations in quantum field theory is a rather challenging field, requiring clever ideas and algorithms for dealing with the difficulties. Instead of presenting a systematic description of the methods used in state-of-the-art computations, here we provide a selection of examples to illustrate the type of ideas that can be helpful in challenging perturbative calculations. More complete descriptions may be found in various review articles, such as refs. 32, 33.

\subsection{Helicity Methods}

As a first example, consider the five-gluon tree amplitude described by Feynman diagrams of which two are depicted in Figure 9. These diagrams are straightforward to evaluate by direct algebraic evaluation on a computer, using one of the algebraic programming languages such as FORM [34, MAPLE, and so forth. The result of evaluating the Feynman diagrams is shown in Figure 10, using a microscopic font in order to illustrate its apparent complexity. The result shown in this figure is actually just the coefficient of one of the color factors; the complete answer is much larger.
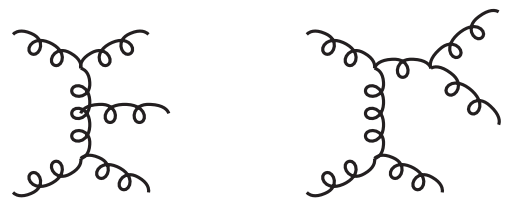

Figure 9. Two of the Feynman diagrams describing the five-gluon tree amplitude.

After some careful thought, a number of authors concluded that the mess of the type shown in Figure 10 is not due to any inherent property of gluon scattering but is only due to using an inefficient representation of the amplitude [35,36]. A much better representation is to use helicity states instead of polarization vectors. For each color a gluon has only two physical states. A polarization vector on the other hand has four components but with two of the degrees of freedom implicitly removed by gauge invariance. It is this mismatch between the number of physical states and the number of components of the polarization vectors that is at the origin of the apparent complexity. A much better representation of the amplitude may be found by making use of helicity or circular polarization. Using its most primitive implementation, one would replace each polarization vector with an explicit circular polarization:

$\varepsilon_{\mu}^{+}=(0,1,+i, 0), \quad \varepsilon_{\mu}^{-}=(0,1,-i, 0)$.

However, this representation is not Lorentz covariant. Much better Lorentz covariant represen- 

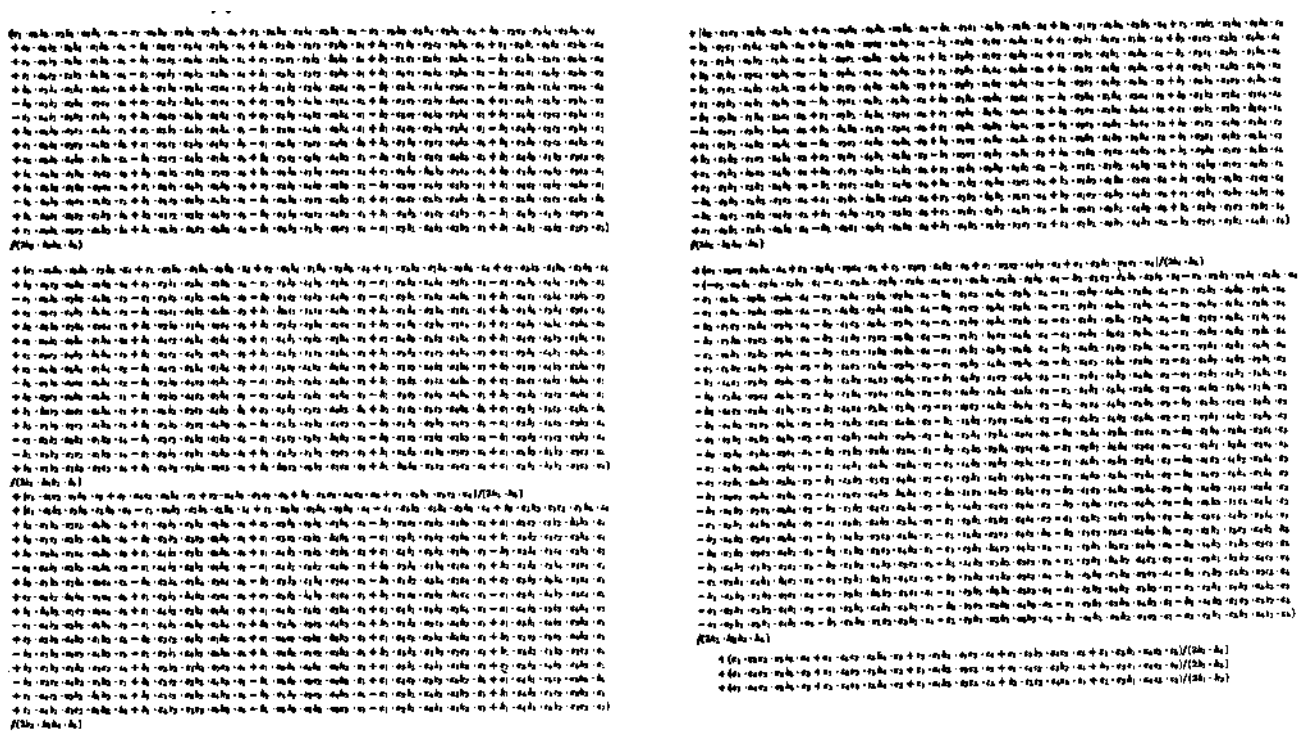

Figure 10. The result of a brute force evaluation of the five-gluon tree amplitude on a computer in an unreadable font. The result shown here is expressed in terms of in terms of dot products of momentum and polarization vectors and is the coefficient of a single color factor.

tations have been invented [35], making use of spinor representations that are more convenient to use. This is sometimes referred to as 'Chinese Magic' in recognition of the especially beautiful formulation due to $\mathrm{Xu}$, Zhang and Chang [36].

In any case, if we make use of helicity, the complete physical content of Figure 10 can instead be written much more neatly in terms of the color stripped helicity amplitudes:

$$
\begin{aligned}
& A_{5}\left(1^{ \pm}, 2^{+}, 3^{+}, 4^{+}, 5^{+}\right)=0 \\
& A_{5}\left(1^{-}, 2^{-}, 3^{+}, 4^{+}, 5^{+}\right)=\left(\frac{s_{12}^{3}}{s_{23} s_{34} s_{45} s_{51}}\right)^{1 / 2}, \\
& A_{5}\left(1^{-}, 2^{+}, 3^{-}, 4^{+}, 5^{+}\right)=\left(\frac{s_{13}^{3}}{s_{23} s_{34} s_{45} s_{51}}\right)^{1 / 2},
\end{aligned}
$$

where the $s_{i j}=\left(p_{i}+p_{j}\right)^{2}$ are the kinematic invariants appearing in the amplitude and the $i$ and $j$ are the labels distinguishing the different gluons. The + and - superscripts for each particle $i$ label the helicity (in a convention where all legs are outgoing).

Given the remarkable cleanup of the ampli- tude, it is perhaps not surprising that helicity representations have allowed seemingly impossible computations to proceed. Perhaps the most famous example of this is the $n$-point generalization of eq. 10 for special helicity configurations, obtained by Parke and Taylor [37]. They conjectured that in QCD the leading order colorstripped $n$-gluon amplitudes satisfy,

$$
\begin{aligned}
& A_{5}\left(1^{ \pm}, 2^{+}, 3^{+}, 4^{+}, \ldots, n+\right)=0 \\
& A_{5}\left(1^{-}, 2^{-}, 3^{+}, 4^{+}, \ldots, n^{+}\right) \\
& \quad=\left(\frac{s_{12}^{3}}{s_{23} s_{34} s_{45} \ldots s_{n 1}}\right)^{1 / 2},
\end{aligned}
$$

for the special helicity configurations indicated by the plus and minus labels. Using standard Feynman rules one would need to evaluate an infinite number of diagrams to obtain this. However, using helicity methods together with recursive techniques, a proof [38] that the Parke-Taylor amplitude are correct was presented soon after the original conjecture. Helicity methods have also been used for constructing infinite sequences of oneloop amplitudes, again for cases of special helicity 
configurations [39,40]. As a more recent example, the first computation of a two-loop $2 \rightarrow 2$ scattering amplitude in QCD [41] also relied on helicity methods.

\subsection{Applications of Supersymmetry}

Supersymmetry has a long history of use in constructing models of possible extensions to the Standard Model. Another application of supersymmetry is providing strong checks on nontrivial calculations in Standard Model physics. The high degree of symmetry renders the dynamics of supersymmetric theories far simpler than those of non-supersymmetric ones used in describing the Standard Model. Calculations in supersymmetric theories can therefore serve as 'toys' for devising methods for dealing with more complicated Standard Model calculations. Moreover a QCD calculation is a close relative of a similar calculation in a supersymmetric version of QCD where the quarks are replaced by gluinos. This allows one to perform checks of QCD calculations by performing minor alternations to the calculations so that they become supersymmetric.

A particularly striking example of the use of supersymmetry as a check is from the computation of the four-loop QCD $\beta$-function performed by van Ritbergen, Vermaseren and Larin [42]. The $\beta$ function is a fundamental quantity controlling the running of the coupling constant. This quantity was already known earlier in a supersymmetric version of QCD 43. However, due to certain technical complications having to do with the different ways that the theories were rendered finite, the two computations could not be compared directly. Nevertheless a non-trivial cancellation of a particular color factor occurs when the QCD result is altered to be supersymmetric, in agreement with the expectation from the supersymmetric version. This suggests that the four-loop calculation of the QCD $\beta$-function is indeed correct. As a more recent example, supersymmetry identities have also been applied as checks on twoloop calculations of scattering amplitudes [11, 144.

\subsection{Quantum Gravity Example of Growth of Difficulty}

It is not difficult to find problems in quantum field theory which cannot possibly be solved by brute force alone. In many cases systematic algorithms for evaluating Feynman diagrams are not known, but even where algorithms are known, problems can easily surpass the capacity of any computer. One example of a particularly interesting problem which possesses this property is the ultraviolet divergences of quantum gravity.

The often-repeated statement that quantum mechanics and General Relativity are incompatible arises from the bad ultra-violet divergences of (super) gravity theories. There are strong arguments that this is in fact correct based on powercounting of the gravity Feynman diagrams (see, e.g., ref. 46]).

For the case of pure Einstein gravity, these arguments were confirmed by an explicit twoloop computation performed by Sagnotti and Goroff 47. However, no proof exists for supergravity theories. The first potential divergence allowed by supergravity occurs at three quantum loops. One of the Feynman diagrams which would need to be evaluated to obtain the divergence is shown in Figure 11. A naive estimate of the total number of terms in this diagram, based on the structure of the propagators and vertices yields approximately $10^{21}$ terms. If one could evaluate terms at the rate of a billion per second, it would take approximately 20,000 years to extract the ultra-violet divergence of just this single diagram. It is no surprise that this computation has not been attempted.

There are, however, methods which can bring this problem into the realm of possible calculations. A trick is to map the gravity calculation in products of gauge theory calculations using certain string theory relations between amplitudes 48.49. The net effect of this is that an impossible problem can be mapped into an extremely difficult problem, which also has not been solved, as yet. This technique has, however, al-

\footnotetext{
${ }^{3}$ There actually is no incompatibility in perturbation theory, up to an energy scale of $10^{19} \mathrm{GeV}$, if General Relativity is viewed as an effective field theory 45 instead of as a fundamental theory.
} 


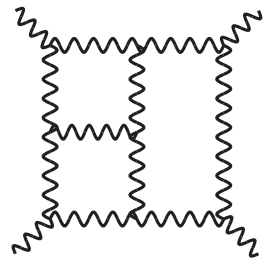

Figure 11. A sample diagram whose divergence part would need to be evaluated in order to determine the ultra-violet divergence of a supergravity theory. The lines represent graviton propagators and the vertices three-graviton interactions.

ready been used to show that at least for the case of maximally supersymmetric gravity the onset of divergences is delayed until at least five quantum loops [19,50].

\section{STATUS OF LOOP CALCULATIONS}

Before surveying the main advance since the last ICHEP conference, it is useful to survey the status of quantum loop calculations. Here we do not discuss tree-level calculations which have also seen considerable progress over the years.

\subsection{Status of one-loop calculations}

In 1948 Schwinger dealt with one-loop threepoint calculations [18] such as that of the anomalous magnetic moment of leptons described in Section 2. It did not take very long before Karplus and Neuman calculated light-bylight scattering in QED in their seminal 1951 paper [51. In 1979 Passarino and Veltman presented the first of many systematic algorithms for dealing with one-loop calculations with up to four external particles, leading to an entire subfield devoted to such calculations. Due to the complexity of non-abelian gauge theories, however, it was not until 1986 that the first purely QCD calculation involving four external partons was carried out in the work of Ellis and Sexton [52.

The first one-loop five-particle scattering amplitude was then calculated in 1993 by Lance Dixon, David Kosower and myself [53] for the case of five-gluon scattering in QCD. This was followed by calculations of the other five-point QCD subprocesses [54], with the associated phys- ical predictions of three-jet events at hadron colliders appearing somewhat later [55,56]. A number of other five-point calculations have also been completed. One example of a state-of-the-art fivepoint calculation was presented in a parallel session by Doreen Wackeroth [57], who described the calculation of $p p \rightarrow \bar{t} t H$ at next-to-leading order in QCD [58]. This process is a useful mode for discovering the Higgs boson as well as measurement of its properties. Other examples are NLO calculations for $e^{+} e^{-} \rightarrow 4$ jets [59,60,61, Higgs +2 jets 62 , and vector boson +2 jet production [59,63], which is also important as a background to the Tevatron Higgs search, if the jets are tagged as coming from $b$ quarks.

Beyond five-external particles, the only calculations have been in special cases. By making use of advanced methods, for special helicity configurations of the particles, infinite sequences of one-loop amplitudes with an arbitrary number of external particles but special helicity configurations have been obtained in a variety of theories [39,40]. For the special case of maximal supersymmetry, six-gluon scattering amplitudes have been obtained for all helicities [40]. There has also been a recent calculation of a six-point amplitude in the Yukawa model [64], as well as recent papers describing properties of six-point integrals [65]. These examples suggest that that the technical know-how for computing general sixpoint amplitudes is available, though it may be a rather formidable task to carry it through. An efficient computer program for dealing with up to three jets at hadron colliders now exists [56], suggesting that it would be possible add one more jet, once the relevant scattering amplitudes are calculated. This would then give a much better theoretical handle on multi-jet production at hadron colliders.

\subsection{Status of Higher Loop Computations}

Over the years, an intensive effort has gone into calculating higher loop Feynman diagrams. A few samples of some impressive multi-loop calculations are:

- The anomalous magnetic moment of leptons, already described in Section 2 . 
- $R=\sigma\left(e^{+} e^{-} \rightarrow\right.$ hadrons $) / \sigma\left(e^{+} e^{-} \rightarrow\right.$ $\left.\mu^{+} \mu^{-}\right)$which has been calculated through $O\left(\alpha_{s}^{3}\right)$ [66]. This quantity is one of the cornerstones demonstrating that $\mathrm{QCD}$ is the correct theory of strong interactions.

- The four-loop QCD $\beta$ function computed by van Ritbergen, Vermaseren and Larin [42]. In the course of this computation approximately 50,000 Feynman diagrams were evaluated, of which a single one is displayed in Figure 12 .

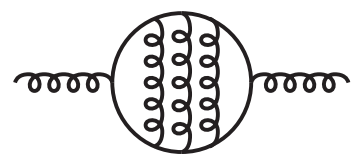

Figure 12. One of the 50,000 Feynman diagrams appearing in the computation of the four-loop QCD $\beta$ function.

These types of precision calculations have been crucial for comparing the Standard Model to experiment 67. However, except for very special cases, all two and higher-loop Feynman diagram computations prior to a few years ago contained either one or no kinematic variables.

\section{ADVANCES OF PAST TWO YEARS}

The key advance in perturbative computations over the last two years is our ability to perform two-loop computations with more than a single kinematic variable. Previously, the only such calculations were for special cases of maximal supersymmetry [68, 49. The pace of progress, due to a large extend to the influx of energetic young people into the field, is such that even though two years ago it was not known how to do such calculations for more general processes, now they are commonplace. A sample diagram illustrating the types of processes that can now be computed is depicted in Figure 13.

Every step in the construction of a physical cross-section involving a two-loop amplitude has

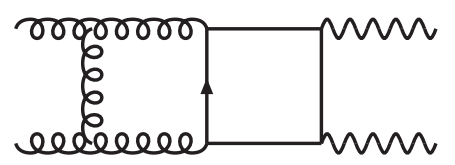

Figure 13. An sample process that has been evaluated. The curly lines represent gluons, the wavy ones photons and the straight ones quarks.

serious technical challenges. Although some of these difficulties have not been completely resolved, all aspects of the problem have seen remarkable progress over the last few years.

\subsection{Loop Integrals}

A crucial ingredient in this progress has been the breakthrough in obtaining the integrals needed for computing two-loop massless $2 \rightarrow 2$ scattering amplitudes 69,70,71, 72. For example, consider the scalar double box integral,

$$
\begin{aligned}
\int & \frac{d^{D} p}{(2 \pi)^{D}} \frac{d^{D} q}{(2 \pi)^{D}} \frac{1}{p^{2} q^{2}(p+q)^{2}\left(p-k_{1}\right)^{2}} \\
& \times \frac{1}{\left(p-k_{1}-k_{2}\right)^{2}\left(q-k_{4}\right)^{2}\left(q-k_{3}-k_{4}\right)^{2}} .
\end{aligned}
$$

which may be represented by the left diagram in Figure 14. This scalar integral was a non-trivial challenge, until it was evaluated by Smirnov 69 in terms of standard polylogarithms. Shortly thereafter, the non-planar scalar integral appearing on the right-hand-side of Figure 14 as well as other relevant integrals were evaluated [70,71]. Moreover, powerful new methods for reducing general tensor integrals into a basis of known integrals were developed [72], making use of integration by parts 73 and Lorentz invariance 74]. A number of important further improvements have also been made allowing for more systematic evaluations 757. The final expressions obtained with these methods are all in terms of standard function such as logarithms, polylogarithms as well as generalizations of these functions that are also amenable to efficient numerical evaluation.

\subsection{Amplitude Calculations}

The first Standard Model calculation of a $2 \rightarrow 2$ scattering amplitude was that of gluon scattering in QCD for a special helicity configuration [41]. By making use of the loop integration break- 

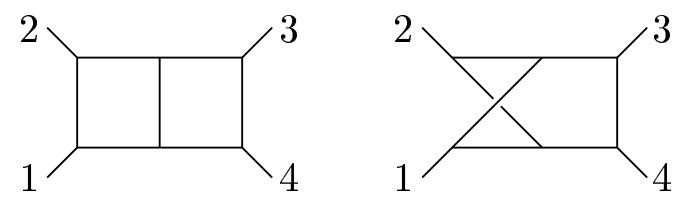

Figure 14. Planar and non-planar double box diagrams.

through, since then many new two-loop amplitudes appeared:

- Bhabha scattering in $\mathrm{QED}, e^{+} e^{-} \rightarrow e^{+} e^{-}$, in the ultra-relativistic limit [14]. Bhabha scattering is useful for monitoring luminosity at an $e^{+} e^{-}$collider.

- All two-loop $2 \rightarrow 2$ QCD processes 10 , 11. These are essential ingredients for constructing NNLO jet programs for hadron colliders.

- Light-by-light scattering, $\gamma \gamma \rightarrow \gamma \gamma$, in the ultra-relativistic limit [76], which is of interest for future photon-photon colliders.

- Gluon fusion into a photon pair, $g g \rightarrow$ $\gamma \gamma$ [8]. As described in Section 6, this subprocess is important as a background to Higgs production when a light Higgs decays into a pair of photons.

- Two loop QED and QCD corrections to massless fermion boson scattering 77]: $\bar{q} q \rightarrow \gamma \gamma, \bar{q} q \rightarrow g \gamma$, and $e^{+} e^{-} \rightarrow \gamma \gamma$.

- $e^{+} e^{-} \rightarrow 3$ partons 12. This is a key process for a future high energy $e^{+} e^{-}$collider and it will allow precision measurements of strong coupling processes at the $1 \%$ level 78 .

- Deeply inelastic scattering 2 jet production and $p p \rightarrow W, Z+1$ jet 12,79 .

As more integrals including ones with massive legs are worked out 80], other amplitudes in QED, electroweak theory, heavy quark physics, etc. will surely also be evaluated. Such calculations are just the initial steps since they are for matrix elements and not for physical predictions. As yet only the $\gamma \gamma \rightarrow \gamma \gamma$ and $g g \rightarrow \gamma \gamma$ amplitudes have been implemented in phenomenological studies [76,9].

\subsection{Infrared Divergences}

The source of much grief in perturbative calculations are infrared divergences. At the end, for any appropriately defined physical quantity these divergences must cancel 81]. However, at intermediate steps of calculations, before the various contributions are combined to form a physically meaningful quantity, severe divergences arise from soft (i.e. $p_{i} \rightarrow 0$ ) or collinear particles. At a given order or perturbation theory, a physical quantity typically involves both virtual and real emission contributions that are separately infrared divergent.

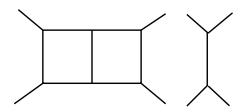

(a)

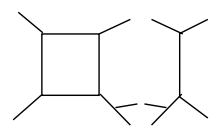

(b)

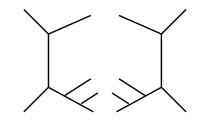

(c)
Figure 15. The various contributions at NNLO to the squared matrix elements: (a) virtual, (b) single real emission and (c) double real emission.

At two-loops it becomes much more difficult to deal with these divergences due to their intricate structure. An important result is Catani's Magic Formula' [82 which specifies the precise form of the two-loop divergences. The 'magic' is that it was obtained before any explicit calculations were possible. Feynman supposedly once remarked that before one calculates one should know the result. Needless to say, this ideal is difficult to achieve, but Catani's Magic Formula is a partial implementation in the sense that the infrared divergences that must emerge are known prior to beginning a calculation. Catani's Magic Formula then provides important guidance in any explicit two loop calculation. It also provides a good way for organizing amplitudes into divergent parts which ultimately drop out of physical 
quantities and the finite contributions which determine the physics.

In order for the advances in computing scattering amplitudes to be used in comparisons of theory to experiments, the various contributions need to be combined into a numerical program for producing the plots comparing theory to experiment. In general, this is non-trivial because of the infrared divergences plaguing intermediate steps of the calculations. At NLO general methods for dealing with the infrared singularities exist [83], but at NNLO as yet there is no complete solution. There is, however, a partial understanding. In particular, the universal behavior of amplitudes entering into NNLO calculations as the momenta of partons become either soft or collinear has been worked out 84,85. Moreover, in certain special cases, such as Drell-Yan [86], inclusive Higgs production at hadron colliders [7, 6] and $e^{+} e^{-} \rightarrow \gamma+1$ jet [87] this has been worked out. It is now clear that more general methods for dealing with the infrared divergences at NNLO will be constructed in the near future.

\subsection{Parton Distribution Functions}

In order to have true NNLO predictions for observables at hadron colliders a crucial ingredient is NNLO parton distribution functions. At NLO the evolution kernels needed for the DGLAP equations [88] were calculated long ago [89]. The calculation of the next order has been a difficult challenge since then, but recent years have seen some impressive progress. The problem is partially solved [90], and very good approximate solutions based on computing Mellin moments have also been obtained 911. These are starting to be implemented in global fits by the MRST group [92]. There is also some related work on NNLO quark and gluon distributions inside photons 933 instead of protons. We can anticipate that it will not be long before all standard parton distribution functions are fully implemented at NNLO. (A description of other important parton distribution function issues, such as uncertainties, may be found in the talks of Giele and Stump [94.)

\subsection{Problems with multiple mass scales.}

For problems with multiple mass scales, in recent years there has also been quite a bit of progress. A powerful general strategy is known as 'the strategy of regions' 95,96, 33. The basic trick makes use of dimensional regularization allowing one to series expand expressions in various kinematic limits or regions without the need for keeping track of the boundaries between regions. The key progress is that now there is a universal method for dealing with this, instead of case-bycase analysis depending on boundaries between the regions. This has been applied to a large number of problems 97], ranging from atomic physics, heavy quark production and decay, large electroweak logarithms and very recently lattice gauge theory. As one example from this conference, the electroweak logarithms described by Kühn [98] were obtained using the strategy of regions.

\subsection{Numerical Methods}

Another area where there has been recent developments is in purely numerical techniques for evaluating Feynman diagrams. An important recent advance in numerical methods was made by Ghinculov and Yao 99], who produced a method for dealing with two-loop problems where the infrared divergences are mild, as typical in problems in electroweak theory. An extension of this was described in the parallel session talk of Passarino 100, targeting state-of-the-art electroweak calculations. In QCD where there are severe infrared divergence, another numerical method, by Binoth and Heinrich [101], has been applied as an important check on state-ofthe-art analytical two-loop calculations. At this conference Dave Soper described a purely numerical method for massless QCD amplitudes [102] So far, this latter method has been used to reproduce the seminal result for $e^{+} e^{-} \rightarrow 3$ jets at NLO obtained by Ellis, Ross and Terrano 103 in 1981, but it is not yet clear if this method will be applied to state-of-the-art calculations. 


\section{SAMPLE APPLICATIONS OF THE BREAKTHROUGH}

In the month prior to the conference, the first two applications of the new advances in two-loop calculation to specific problems of interest for collider physics appeared 9.6]. These first examples focus on Higgs physics in part because of its importance for the collider physics program, but also because the infrared singularities for these cases are relatively straightforward to handle. Once the general problem of infrared divergent phase space integrals is solved, many more applications will appear.

The first of these applications provides an improved understanding of the QCD background to Higgs production and decay for the case of a light Higgs. The second application, which appeared just two weeks prior to the conference, provides the exact result for inclusive Higgs production [6] at NNLO. This result is in perfect agreement with an earlier series expansion due to Harlander and Kilgore [7] and in very good agreement with an even earlier approximate solution 104.105.

\subsection{Inclusive Higgs Production}

In his talk, Frixione [13] described the important quest to obtain reliable predictions for Higgs production at the Tevatron and LHC. Previously, inclusive Higgs production had been calculated through NLO 106, leaving a substantial theoretical uncertainty in the result. The new NNLO calculations significantly reduce the theoretical uncertainties.

Because of the large Yukawa coupling of the Higgs to top quarks and the fact that the LHC acts as a glue factory the dominant Higgs production mechanism LHC is though gluon fusion via a top loop. For a light mass Higgs boson, preferred by precision electroweak measurements, the top loop can me replaced by a simpler effective vertex 107, as depicted in Figure 16. In this case, the effective vertex is an excellent approximation 108.

Using the effective Higgs vertex, the two-loop virtual contributions of the type in Figure 17(a) were worked out recently by Harlander 109. The especially difficult part of the calculation is deal-

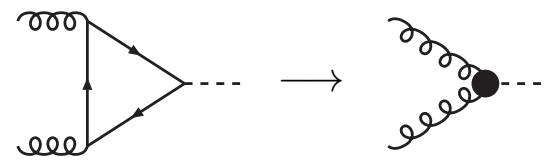

Figure 16. For a light Higgs the top quark loop can be replaced by an effective vertex. The curly lines represent gluons, the solid ones the top quark, and the dashed ones the Higgs boson.

ing with the IR singular integration over the double real emission phase space encountered when evaluating contributions arising from diagrams of the type shown in Figure 17(c). This was solved recently by Harlander and Kilgore [7] who constructed a series expansion of the NNLO inclusive cross section.

The next advance was by Anastasiou and Melnikov who applied the new loop integration technology outlined in Section 5.1 to obtain the exact phase space integration over the various contributions including the double real emission ones [6]. The essential trick for doing this is based on unitarity, as encoded in the Cutkosky cutting rules 110$]$. The relationship between phase space and Feynman loop integrals is a rather useful trick, having, for example, also been used to obtain a number of two-loop scattering amplitudes 41, 3,11]. With this trick it is possible to replace the phase space integration, including those for double real emission, with standard Feynman loop integrals. Once the problem is expressed in terms of standard Feynman integrals the new integration methods can be applied directly. So far this trick has been applied to totally inclusive cross-sections, but it is very likely that it can be extended to at least certain differential crosssections 111.

The basic result of both these calculations is summarized in Figure 18, obtained from ref. [7]. The perturbative expansion clearly stabilizes at NNLO and is reliable, given the modest shift between the NLO and NNLO results. Further refinements to the NNLO results should also be forthcoming [105. 


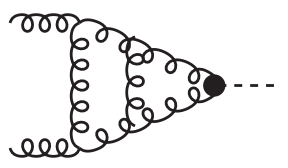

(a)

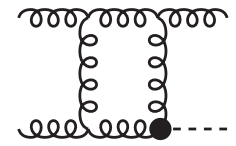

(b)

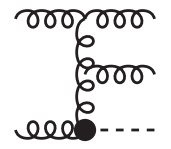

(c)

Figure 17. Sample diagrams representing (a) virtual, (b) single emission and (c) double emission contributions. The curly lines represent gluons, the dashed ones Higgs bosons, and the solid circle the effective vertex coupling gluons to the Higgs.

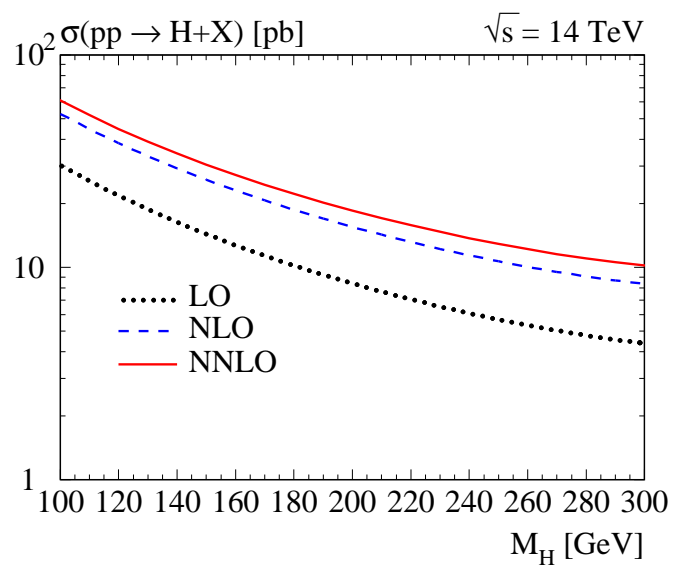

Figure 18. The LO, NLO and NNLO predictions for inclusive Higgs productions at the LHC as a functions of the Higgs mass (with factorization and renormalization scales set equation to the Higgs mass).

\subsection{The Di-Photon Background to Higgs Decay}

The second application of the breakthrough that we describe is for the background to observing a light $\left(M_{H}<140 \mathrm{GeV}\right)$ Higgs boson at the LHC. For a light Higgs, a key search mode at the LHC involves Higgs production via gluon fusion followed by the rare decay into a pair of photons. (For a recent survey of ways to detect a light Higgs boson at the LHC see, for example, ref. [112].) Although the branching ratio is tiny, this mode is relatively clean due to the excellent mass resolution of the LHC detectors. This allows the background to be measured experimentally and subtracted from a putative sig- nal 113 114,115. Nevertheless, it is still important to have robust theoretical predictions in order to systematically study the dependence of the signal relative to the background so as to optimize Higgs search strategies. Given that for the case of a light Higgs boson it will take about two years of running at the LHC before the Higgs signal is pulled out of the background, there is good motivation for wanting to optimize search strategies.

The background is composed of two pieces. The 'reducible' background is where photons are faked by jets, or more generally by hadrons, especially $\pi^{0} \mathrm{~s}$. This background can be suppressed efficiently by photon isolation cuts, where events are rejected based on the amount of hadronic energy near the photons. The second source of background is from the underlying QCD process where quarks emit photons either directly or through fragmentation. The process $p p \rightarrow \gamma \gamma X$ proceeds at lowest order via the quark annihilation subprocess $q \bar{q} \rightarrow \gamma \gamma$, which is independent of the strong coupling $\alpha_{s}$. One of the Feynman diagrams describing this process is shown in Figure 19. The next-to-leading-order (NLO) corrections to this subprocess have been incorporated into a number of Monte Carlo programs [116], the most up-todate being DIPHOX 117.

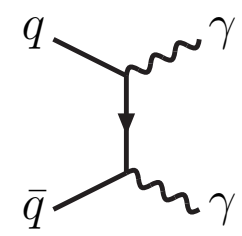

Figure 19. A leading order diagram contributing to di-photon production. 
Although the gluon fusion contribution depicted in Figure 20 is formally of higher order in the QCD coupling, it is enormously enhanced by the fact that the distribution in the proton becomes very large at small $x$. This makes formally higher order corrections involving gluon initial states very significant for the production of low-mass systems $(<200 \mathrm{GeV})$ at the LHC. The net result is that the gluon fusion contribution to $p p \rightarrow \gamma \gamma X$ is comparable to the leading-order quark annihilation contribution [116, 117, 118, 119.

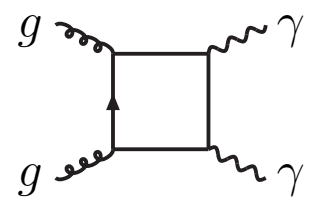

Figure 20. A leading order diagram contributing to the gluon fusion into two photons.

To reduce the uncertainty on the total $\gamma \gamma$ production rate, one therefore needs to calculate the $g g \rightarrow \gamma \gamma$ subprocess at its next-to-leading-order, even though it is formally $\mathrm{N}^{3} \mathrm{LO}$ as far as the whole process $p p \rightarrow \gamma \gamma X$ is concerned. This involves the diagram of the type shown in Figure 21. The two-loop virtual contributions (a) were computed recently in ref. [8], while the one-loop real emission contribution (b) is obtained from a permutation sum [120] over contributions to the oneloop five-gluon amplitude [53].

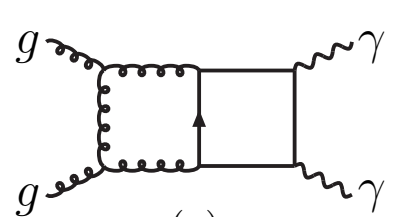

(a)

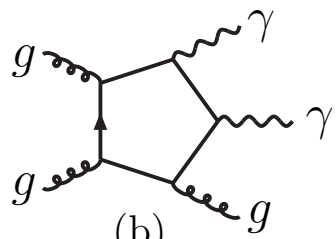

(b)
Figure 21. Sample NLO diagrams contributing to gluon fusion into two photons: (a) virtual and (b) real emission contributions.

To obtain the physical cross-section the two- loop contributions of the type in Figure 21(a) must be combined with the real emission contributions in Figure 21(b) in such a way that all infrared singularities cancel. In this case the infrared singularities are all in a form which can be handled using standard NLO methods 83]. Then these new contributions must be combined with the previously obtained ones 117].

A sample result taken from ref. [9] is shown in Figure 22. The conclusion from this plot is that in the region of interest the NLO corrections to the $g g \rightarrow \gamma \gamma$ subprocess have a relatively modest effect on the total irreducible di-photon background to the Higgs search. Indeed the $K$ factor (ratio of NLO to LO contributions) for this subprocess is significantly smaller than previous estimates somewhat enhancing the statistical significance of the Higgs signal compared to previous estimates. Moreover, the relatively small shift from LO to NLO suggests that the subprocess is under adequate theoretical control 9 .

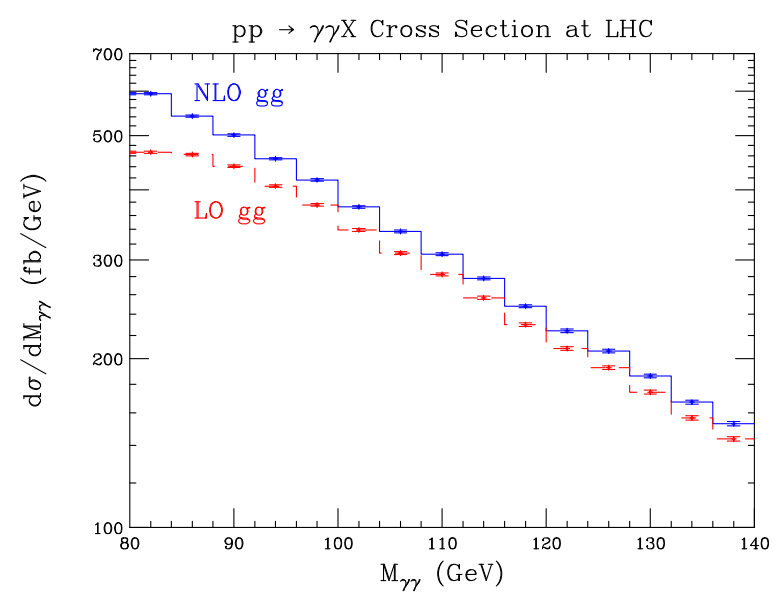

Figure 22. The $p p \rightarrow \gamma \gamma X$ cross-section as a function of invariant mass of the di-photon pair at the LHC. The lower curve is obtained using DIPHOX. The upper curve is the result of adding in the extra NLO contributions to gluon fusion.

A crucial question is whether we can improve the situation by finding appropriate search strate- 
gies which enhance the signal over the background. Some initial studies of this question at the parton level were performed in ref. [9], but more realistic studies are required, including detector effects 115 and the reducible $\pi^{0}$ background contributions 114,115,121.

\section{SUMMARY AND OUTLOOK}

A very recent example illustrating the importance of precision perturbative calculations is the impressive measurement of the anomalous magnetic moment of the muon by the Brookhaven $g-2$ collaboration. The first part of this talk outlined the intense theoretical effort that has gone into obtaining the Standard Model prediction at a precision matching the one of the experiment.

This talk also highlighted the recent rapid pace of progress in our ability to compute quantities of interest in perturbative quantum field theory. In particular, two-loop calculations involving up to four external particles can now be performed in rather general cases. This breakthrough is the culmination of years of effort. Parallel advances in calculations of the DGLAP evolution of parton distribution functions have also been made.

So far the breakthrough had been applied mainly to QCD and to massless QED, but as more types of loop integrals with masses are calculated one can expect applications also to electroweak theory, heavy quark physics, QED with massive particles, and so forth. Two very recent phenomenological applications of the advance are the calculation of the exact next-to-next-to-leading order inclusive Higgs production cross-section at hadron colliders and the calculation of the QCD background to Higgs production and decay into a photon pair at the LHC, for the case of a light Higgs. Once general algorithms are set up for dealing with infrared divergent phase space at next-to-next-leading order, many more phenomenological studies will appear.

Given the influx into the field of the many talented energetic young people who have contributed greatly to the advances described here, we can be optimistic that the rapid pace of progress will continue for the foreseeable future.

\section{Acknowledgments}

I thank Lance Dixon and David Kosower for their many thoughtful comments and contributions. I also thank Bill Marciano for a number of very illuminating discussions on the status of the anomalous magnetic moment of the muon. Finally, I thank Stefano Catani, Abilio De Freitas, Einan Gardi, Bill Kilgore, Kirill Melnikov, Carl Schmidt, and Henry Wong, as well as all the people who have made the recent advances possible.

\section{REFERENCES}

1. G. Degrassi, arXiv:hep-ph/0102137; J. Erler, arXiv:hep-ph/0102143; D. Abbaneo et al. [ALEPH, DELPHI, L3 and OPAL Collaborations, LEP Electroweak Working Group, and SLD Heavy Flavor and Electroweak Groups], arXiv:hep-ex/0112021.

2. H. N. Brown et al. [Muon $(g-2)$ Collaboration], Phys. Rev. Lett. 86, 2227 (2001) arXiv:hep-ex/0102017.

3. G. W. Bennett et al. [Muon $(g-2)$ Collaboration], Phys. Rev. Lett. 89, 101804 (2002) [Erratum-ibid. 89, 129903 (2002)] arXiv:hepex/0208001].

4. Y. K. Semertzidis [Muon $(g-2)$ collaboration], in these proceedings arXiv:hep$\mathrm{ph} / 0211038$.

5. T. Teubner, in these proceedings.

6. C. Anastasiou and K. Melnikov, arXiv:hep$\mathrm{ph} / 0207004$.

7. R. V. Harlander and W. B. Kilgore, Phys. Rev. Lett. 88, 201801 (2002) arXiv:hepph/0201206|; W. B. Kilgore, in these proceedings [arXiv:hep-ph/0208143].

8. Z. Bern, A. De Freitas and L. J. Dixon, JHEP 0109, 037 (2001) arXiv:hep-ph/0109078.

9. Z. Bern, L. Dixon and C. Schmidt, arXiv:hepph/0206194, to appear in Phys. Rev. D.

10. C. Anastasiou, E.W. Glover, C. Oleari and M.E. Tejeda-Yeomans, Nucl. Phys. B 601, 318 (2001) hep-ph/0010212]; C. Anastasiou, E.W. Glover, C. Oleari and M.E. TejedaYeomans, Nucl. Phys. B 601, 341 (2001) hepph/0011094]; C. Anastasiou, E.W. Glover, C. Oleari and M.E. Tejeda-Yeomans, Nucl. 
Phys. B 605, 486 (2001) hep-ph/0101304; E.W. Glover, C. Oleari and M.E. TejedaYeomans, Nucl. Phys. B 605, 467 (2001).

11. Z. Bern, A. De Freitas and L. Dixon, JHEP 0203, 018 (2002) arXiv:hep-ph/0201161.

12. L. W. Garland, T. Gehrmann, E. W. Glover, A. Koukoutsakis and E. Remiddi, Nucl. Phys. B 627, 107 (2002) arXiv:hep-ph/0112081; L. W. Garland, T. Gehrmann, E. W. Glover, A. Koukoutsakis and E. Remiddi, Nucl. Phys. B 642, 227 (2002) arXiv:hep-ph/0206067; S. Moch, P. Uwer and S. Weinzierl, arXiv:hep-ph/0207043; S. Moch, P. Uwer and S. Weinzierl, arXiv:hep-ph/0210009,

13. S. Frixione, in these proceedings arXiv:hep$\mathrm{ph} / 0211434$.

14. Z. Bern, L. Dixon and A. Ghinculov, Phys. Rev. D 63, 053007 (2001) [hep-ph/0010075].

15. E. W. Glover, J. B. Tausk and J. J. Van der Bij, Phys. Lett. B 516, 33 (2001) arXiv:hep$\mathrm{ph} / 0106052$.

16. A. Czarnecki and W. J. Marciano, Nucl. Phys. Proc. Suppl. 76, 245 (1999) arXiv:hepph/9810512]; A. Czarnecki and W. J. Marciano, in Proc. of the 5th International Symposium on Radiative Corrections (RADCOR 2000) ed. Howard E. Haber, arXiv:hep$\mathrm{ph} / 0010194$.

17. A. Czarnecki and W. J. Marciano, Phys. Rev. D 64, 013014 (2001) arXiv:hep-ph/0102122.

18. J. S. Schwinger, Phys. Rev. 73, 416 (1948).

19. F. Jegerlehner, arXiv:hep-ph/0104304, W. J. Marciano and B. L. Roberts, arXiv:hep$\mathrm{ph} / 0105056$.

20. C. M. Sommerfield, Phys. Rev. 107, 328 (1957); Ann. Phys. 5, 26 (1958); A. Petermann, Nucl. Phys. 3, 689 (1957); Helv. Phys. Acta 30, 407 (1957); T. Kinoshita, Z. Phys. C 56, S80 (1992); V. W. Hughes and T. Kinoshita, Rev. Mod. Phys. 71, S133 (1999).

21. T. Kinoshita, B. Nizic and Y. Okamoto, Phys. Rev. D 41, 593 (1990).

22. S. Laporta and E. Remiddi, Phys. Lett. B379, 283 (1996).

23. G. Li, R. Mendel, and M. A. Samuel, Phys. Rev. D47, 1723 (1993); H. H. Elend, Phys. Lett. 20, 682 (1966), erratum: ibid., vol. 21, p. 720 ; S. Laporta and E. Remiddi, Phys.
Lett. B301, 440 (1993); B. Krause, Phys. Lett. B390, 392 (1997).

24. T. Kinoshita and M. Nio, arXiv:hep$\mathrm{ph} / 0210322$.

25. T. Kinoshita, B. Nizic, and Y. Okamoto, Phys. Rev. D41, 593 (1990); A. S. Yelkhovsky, Sov. J. Nucl. Phys. 49, 656 (1989); A. I. Milstein and A. S. Yelkhovsky, Phys. Lett. B233, 11 (1989); S. G. Karshenboim, Phys. Atom. Nucl. 56, 857 (1993); A. L. Kataev, Phys. Lett. B284, 401 (1992); A. L. Kataev and V. V. Starshenko, Phys. Rev. D52, 402 (1995); J. Ellis, M. Karliner, M. A. Samuel, and E. Steinfelds, hep$\mathrm{ph} / 9409376$.

26. K. Fujikawa, B. W. Lee, and A. I. Sanda, Phys. Rev. D6, 2923 (1972); R. Jackiw and S. Weinberg, Phys. Rev. D5, 2473 (1972); G. Altarelli, N. Cabibbo, and L. Maiani, Phys. Lett. B40, 415 (1972); I. Bars and M. Yoshimura, Phys. Rev. D6, 374 (1972); W. A. Bardeen, R. Gastmans, and B. E. Lautrup, Nucl. Phys. B46, 315 (1972).

27. A. Czarnecki, B. Krause, and W. Marciano, Phys. Rev. D52, R2619 (1995); A. Czarnecki, B. Krause, and W. Marciano, Phys. Rev. Lett. 76, 3267 (1996); T. V. Kukhto, E. A. Kuraev, A. Schiller, and Z. K. Silagadze, Nucl. Phys. B371, 567 (1992); S. Peris, M. Perrottet, and E. de Rafael, Phys. Lett. B355, 523 (1995).

28. M. Gourdin and E. De Rafael, Nucl. Phys. B 10 (1969) 667; S. Eidelman and F. Jegerlehner, Z. Phys. C 67, 585 (1995) arXiv:hep-ph/9502298.

29. T. Kinoshita, B. Nizic and Y. Okamoto, Phys. Rev. D 31, 2108 (1985); M. Hayakawa, T. Kinoshita and A. I. Sanda, Phys. Rev. Lett. 75, 790 (1995) arXiv:hep-ph/9503463; J. Bijnens, E. Pallante and J. Prades, Nucl. Phys. B 474, 379 (1996) arXiv:hepph/9511388]; M. Hayakawa, T. Kinoshita and A. I. Sanda, Phys. Rev. D 54, 3137 (1996) arXiv:hep-ph/9601310; E. Bartos, A. Z. Dubnickova, S. Dubnicka, E. A. Kuraev and E. Zemlyanaya, Nucl. Phys. B 632, 330 (2002) arXiv:hep-ph/0106084.

30. M. Knecht and A. Nyffeler, Phys. Rev. D 65, 
073034 (2002) arXiv:hep-ph/0111058.

31. E. de Rafael, arXiv:hep-ph/0208251; S. I. Eidelman, S. G. Karshenboim and V. A. Shelyuto, arXiv:hep-ph/0209146; K. Hagiwara, A. D. Martin, D. Nomura and T. Teubner, arXiv:hep-ph/0209187.

32. M.L. Mangano and S.J. Parke, Phys. Rept. 200, 301 (1991); L. Dixon, in Proceedings of Theoretical Advanced Study Institute in Elementary Particle Physics (TASI 95), ed. D.E. Soper hep-ph/9601359; Z. Bern, L. J. Dixon and D. A. Kosower, Ann. Rev. Nucl. Part. Sci. 46, 109 (1996) arXiv:hep-ph/9602280; M. Steinhauser, Phys. Rept. 364, 247 (2002) arXiv:hep-ph/0201075.

33. V. A. Smirnov, Applied Asymptotic Expansions In Momenta And Masses, Berlin, Germany: Springer (2002) $262 \mathrm{p}$.

34. J. A. Vermaseren, KEK-TH-326.

35. F.A. Berends, R. Kleiss, P. De Causmaecker, R. Gastmans and T.T. Wu, Phys. Lett. B 103, 124 (1981); P. De Causmaecker, R. Gastmans, W. Troost and T.T. Wu, Phys. Lett. B 105, 215 (1981).

36. Z. Xu, D. Zhang and L. Chang, Nucl. Phys. B 291, 392 (1987).

37. S. J. Parke and T. R. Taylor, Phys. Rev. Lett. 56, 2459 (1986).

38. F. A. Berends and W. T. Giele, Nucl. Phys. B 306, 759 (1988); D. A. Kosower, Nucl. Phys. B 335, 23 (1990).

39. G. Mahlon, Phys. Rev. D 49, 4438 (1994) arXiv:hep-ph/9312276]; Phys. Rev. D 49, 2197 (1994) arXiv:hep-ph/9311213]; Z. Bern, G. Chalmers, L. J. Dixon and D. A. Kosower, Phys. Rev. Lett. 72, 2134 (1994) arXiv:hepph/9312333; Z. Bern, L. J. Dixon, D. C. Dunbar and D. A. Kosower, Nucl. Phys. B 425, 217 (1994) arXiv:hep-ph/9403226; Z. Bern, L. J. Dixon, M. Perelstein and J. S. Rozowsky, Nucl. Phys. B 546, 423 (1999) arXiv:hepth/9811140]; Z. Bern, L. J. Dixon, M. Perelstein and J. S. Rozowsky, Phys. Lett. B 444, 273 (1998) hep-th/9809160; Z. Bern, L. J. Dixon, M. Perelstein and J. S. Rozowsky, Nucl. Phys. B 546, 423 (1999). hepth/9811140.

40. Z. Bern, L. J. Dixon, D. C. Dunbar and
D. A. Kosower, Nucl. Phys. B 435, 59 (1995) arXiv:hep-ph/9409265.

41. Z. Bern, L. Dixon and D.A. Kosower, JHEP 0001, 027 (2000) hep-ph/0001001.

42. T. van Ritbergen, J. A. Vermaseren and S. A. Larin, Phys. Lett. B 400, 379 (1997).

43. I. Jack, D. R. Jones and C. G. North, Nucl. Phys. B 486, 479 (1997) arXiv:hep$\mathrm{ph} / 9609325$.

44. T. Binoth, E. W. Glover, P. Marquard and J. J. van der Bij, JHEP 0205, 060 (2002) arXiv:hep-ph/0202266; Z Z. Bern, A. De Freitas, L. Dixon and H. L. Wong, arXiv:hepph/0202271, to appear in Phys. Rev. D.

45. J. F. Donoghue, Phys. Rev. D 50, 3874 (1994) arXiv:gr-qc/9405057.

46. P. S. Howe and K. S. Stelle, Int. J. Mod. Phys. A 4, 1871 (1989); P. S. Howe and K. S. Stelle, arXiv:hep-th/0211279.

47. M. H. Goroff and A. Sagnotti, Nucl. Phys. B 266, 709 (1986); A. E. van de Ven, Nucl. Phys. B 378, 309 (1992).

48. H. Kawai, D. C. Lewellen and S. H. Tye, Nucl. Phys. B 269, 1 (1986).

49. Z. Bern, L. Dixon, D. C. Dunbar, M. Perelstein and J. S. Rozowsky, Nucl. Phys. B 530, 401 (1998) hep-th/9802162.

50. K. S. Stelle, preprint hep-th/0203015.

51. R. Karplus and M. Neuman, Phys. Rev. 83, 776 (1951).

52. R. K. Ellis and J. C. Sexton, Nucl. Phys. B 269, 445 (1986).

53. Z. Bern, L. J. Dixon and D. A. Kosower, Phys. Rev. Lett. 70, 2677 (1993) arXiv:hep$\mathrm{ph} / 9302280$.

54. Z. Kunszt, A. Signer and Z. Trocsanyi, Phys. Lett. B 336, 529 (1994) arXiv:hepph/9405386]; Z. Bern, L. J. Dixon and D. A. Kosower, Nucl. Phys. B 437, 259 (1995) arXiv:hep-ph/9409393.

55. W. B. Kilgore and W. T. Giele, Phys. Rev. D 55, 7183 (1997) arXiv:hep-ph/9610433.

56. Z. Nagy, Phys. Rev. Lett. 88, 122003 (2002) arXiv:hep-ph/0110315].

57. S. Dawson, L. Orr, L. Reina and D. Wackeroth, in these proceedings arXiv:hep-ph/0210109.

58. L. Reina and S. Dawson, Phys. Rev. Lett. 
87, 201804 (2001) arXiv:hep-ph/0107101; W. Beenakker, S. Dittmaier, M. Kramer, B. Plumper, M. Spira and P. M. Zerwas, Phys. Rev. Lett. 87, 201805 (2001) arXiv:hep-ph/0107081; L. Reina, S. Dawson and D. Wackeroth, Phys. Rev. D 65, 053017 (2002) arXiv:hep-ph/0109066.

59. Z. Bern, L. J. Dixon, D. A. Kosower and S. Weinzierl, Nucl. Phys. B 489, 3 (1997) arXiv:hep-ph/9610370; Z. Bern, L. J. Dixon and D. A. Kosower, Nucl. Phys. B 513, 3 (1998) arXiv:hep-ph/9708239.

60. E. W. Glover and D. J. Miller, Phys. Lett. B 396, 257 (1997) arXiv:hep-ph/9609474; J. M. Campbell, E. W. Glover and D. J. Miller, Phys. Lett. B 409, 503 (1997) arXiv:hep-ph/9706297.

61. A. Signer and L. J. Dixon, Phys. Rev. Lett. 78, 811 (1997) arXiv:hep-ph/9609460]; Phys. Rev. D 56, 4031 (1997) |arXiv:hepph/9706285]; Z. Nagy and Z. Trocsanyi, Phys. Rev. D 59, 014020 (1999) [Erratumibid. D 62, 099902 (2000) arXiv:hepph/9806317]; J. M. Campbell, M. A. Cullen and E. W. Glover, Eur. Phys. J. C 9, 245 (1999) arXiv:hep-ph/9809429]; S. Weinzierl and D. A. Kosower, Phys. Rev. D 60, 054028 (1999) arXiv:hep-ph/9901277.

62. V. Del Duca, W. Kilgore, C. Oleari, C. Schmidt and D. Zeppenfeld, Nucl. Phys. B 616, 367 (2001) arXiv:hep-ph/0108030.

63. R. K. Ellis and S. Veseli, Phys. Rev. D 60, 011501 (1999) arXiv:hep-ph/9810489; J. M. Campbell and R. K. Ellis, Phys. Rev. D 62, 114012 (2000) arXiv:hep-ph/0006304; J. Campbell and R. K. Ellis, Phys. Rev. D 65, 113007 (2002) arXiv:hep-ph/0202176.

64. T. Binoth, J. P. Guillet, G. Heinrich and C. Schubert, Nucl. Phys. B 615, 385 (2001) arXiv:hep-ph/0106243.

65. T. Binoth, J. P. Guillet and G. Heinrich, Nucl. Phys. B 572, 361 (2000) arXiv:hepph/9911342 ; T. Binoth, G. Heinrich and N. Kauer, arXiv:hep-ph/0210023; A. Ferroglia, M. Passera, G. Passarino and S. Uccirati, arXiv:hep-ph/0209219; A. T. Suzuki, E. S. Santos and A. G. Schmidt, arXiv:hep$\mathrm{ph} / 0210083$.
66. S. G. Gorishnii, A. L. Kataev and S. A. Larin, Phys. Lett. B 259, 144 (1991). S. G. Gorishnii, A. L. Kataev and S. A. Larin, JETP Lett. 53, 127 (1991) [Pisma Zh. Eksp. Teor. Fiz. 53, 121 (1991)]; L. R. Surguladze and M. A. Samuel, Phys. Rev. Lett. 66, 560 (1991) [Erratum-ibid. 66, 2416 (1991)].

67. K. Hagiwara et al. [Particle Data Group Collaboration], Phys. Rev. D 66, 010001 (2002).

68. Z. Bern, J.S. Rozowsky and B. Yan, Phys. Lett. B 401, 273 (1997) hep-ph/9702424.

69. V.A. Smirnov, Phys. Lett. B460, 397 (1999) hep-ph/9905323.

70. J.B. Tausk, Phys. Lett. B469, 225 (1999) hep-ph/9909506.

71. C. Anastasiou, E.W.N. Glover and C. Oleari, Nucl. Phys. B565, 445 (2000) hepph/9907523]; C. Anastasiou, E.W.N. Glover and C. Oleari, Nucl. Phys. B575, 416 (2000), err. ibid. B585, 763 (2000) hep-ph/9912251; T. Gehrmann and E. Remiddi, Nucl. Phys. B 601, 248 (2001) arXiv:hep-ph/0008287; T. Gehrmann and E. Remiddi, Nucl. Phys. B 601, 287 (2001) arXiv:hep-ph/0101124.

72. V.A. Smirnov and O.L. Veretin, Nucl. Phys. B566, 469 (2000) hep-ph/9907385; C. Anastasiou, T. Gehrmann, C. Oleari, E. Remiddi and J.B. Tausk, Nucl. Phys. B580, 577 (2000) hep-ph/0003261].

73. F. V. Tkachov, Phys. Lett. B 100, 65 (1981); K. G. Chetyrkin and F. V. Tkachov, Nucl. Phys. B 192, 159 (1981).

74. T. Gehrmann and E. Remiddi, Nucl. Phys. B580, 485 (2000) hep-ph/9912329.

75. S. Laporta, Int. J. Mod. Phys. A 15, 5087 (2000) arXiv:hep-ph/0102033; S. Moch, P. Uwer and S. Weinzierl, J. Math. Phys. 43, 3363 (2002) arXiv:hep-ph/0110083.

76. Z. Bern, A. De Freitas, L. J. Dixon, A. Ghinculov and H. L. Wong, JHEP 0111, 031 (2001) arXiv:hep-ph/0109079.

77. C. Anastasiou, E. W. Glover and M. E. Tejeda-Yeomans, Nucl. Phys. B 629, 255 (2002) arXiv:hep-ph/0201274.

78. P. N. Burrows et al., arXiv:hep-ex/9612012.

79. T. Gehrmann and E. Remiddi, Nucl. Phys. B 640, 379 (2002) arXiv:hep-ph/0207020.

80. V. A. Smirnov, Phys. Lett. B 524, 129 (2002) 
|arXiv:hep-ph/0111160|.

81. T. Kinoshita, J. Math. Phys. 3, 650 (1962); T. D. Lee and M. Nauenberg, Phys. Rev. 133, B1549 (1964).

82. S. Catani, Phys. Lett. B 427, 161 (1998) arXiv:hep-ph/9802439].

83. W. T. Giele and E. W. Glover, Phys. Rev. D 46, 1980 (1992); W. T. Giele, E. W. Glover and D. A. Kosower, Nucl. Phys. B 403, 633 (1993) arXiv:hep-ph/9302225; S. Frixione, Z. Kunszt and A. Signer, Nucl. Phys. B 467, 399 (1996) arXiv:hep-ph/9512328]; S. Catani and M. H. Seymour, Nucl. Phys. B 485, 291 (1997) [Erratum-ibid. B 510, 503 (1997)] arXiv:hep-ph/9605323.

84. Z. Bern and G. Chalmers, Nucl. Phys. B 447, 465 (1995) arXiv:hep-ph/9503236; Z. Bern, V. Del Duca and C. R. Schmidt, Phys. Lett. B 445, 168 (1998) arXiv:hep-ph/9810409; D. A. Kosower and P. Uwer, Nucl. Phys. B 563, 477 (1999) arXiv:hep-ph/9903515; Z. Bern, V. Del Duca, W. B. Kilgore and C. R. Schmidt, Phys. Rev. D 60, 116001 (1999) arXiv:hep-ph/9903516]; S. Catani and M. Grazzini, Nucl. Phys. B 591, 435 (2000) arXiv:hep-ph/0007142.

85. J.M. Campbell and E.W.N. Glover, Nucl. Phys. B527, 264 (1998) hep-ph/9710255; S. Catani and M. Grazzini, Phys. Lett. B446, 143 (1999) hep-ph/9810389; S. Catani and M. Grazzini, Nucl. Phys. B 570, 287 (2000) arXiv:hep-ph/9908523].

86. R. Hamberg, W. L. van Neerven and T. Matsuura, Nucl. Phys. B 359, 343 (1991).

87. A. Gehrmann-De Ridder and E. W. Glover, Nucl. Phys. B 517, 269 (1998) arXiv:hep$\mathrm{ph} / 9707224$.

88. Yu. L. Dokshitzer, Sov. Phys. JETP 46 (1977) 641; V.N. Gribov and L.N. Lipatov, Sov. J. Nucl. Phys. 15 (1972) 675; G. Altarelli and G. Parisi, Nucl. Phys. B126 (1977) 298.

89. G. Curci, W. Furmanski and R. Petronzio, Nucl. Phys. B 175, 27 (1980).

90. E. B. Zijlstra and W. L. van Neerven, Nucl. Phys. B 383, 525 (1992). S. Moch and J.A.M. Vermaseren, Nucl. Phys. (Proc. Suppl.) 89 (200) 131, 137; S. Moch, J. A. Vermaseren and M. Zhou, arXiv:hep- ph/0108033; S. Moch, J. A. Vermaseren and A. Vogt, preprint arXiv:hep-ph/0209100.

91. S.A. Larin, T. van Ritbergen, and J.A.M. Vermaseren, Nucl. Phys. B427 (1994) 41; S.A. Larin, P. Nogueira, T. van Ritbergen, and J.A.M. Vermaseren, Nucl. Phys. B492 (1997) 338 S. Catani and F. Hautmann, Nucl. Phys. B427 (1994) 475; J. Blümlein and A. Vogt, Phys. Lett. B370 1996) 149; V.S. Fadin and L.N. Lipatov, Phys. Lett. B429 (1998) 127; M. Ciafaloni and G. Camici, Phys. Lett. B430 (1998) 349; W. L. van Neerven and A. Vogt, Phys. Lett. B 490, 111 (2000) arXiv:hep-ph/0007362]; W. L. van Neerven and A. Vogt, Nucl. Phys. B 568, 263 (2000) arXiv:hep-ph/9907472; W. L. van Neerven and A. Vogt, Nucl. Phys. B 588, 345 (2000) arXiv:hep-ph/0006154; A. Retey and J. A. Vermaseren, Nucl. Phys. B 604, 281 (2001) arXiv:hep-ph/0007294.

92. A. D. Martin, R. G. Roberts, W. J. Stirling and R. S. Thorne, Phys. Lett. B 531, 216 (2002) arXiv:hep-ph/0201127.

93. S. Moch, J. A. Vermaseren and A. Vogt, Nucl. Phys. B 621, 413 (2002) arXiv:hep$\mathrm{ph} / 0110331$.

94. W. Giele, in these proceedings; Daniel R. Stump, in these proceedings.

95. S. G. Gorishnii, Nucl. Phys. B 319, 633 (1989); K. G. Chetyrkin, Theor. Math. Phys. 75, 346 (1988) [Teor. Mat. Fiz. 75, 26 (1988)]; V. A. Smirnov, Commun. Math. Phys. 134, 109 (1990).

96. F. V. Tkachov, Phys. Lett. B 412, 350 (1997) arXiv:hep-ph/9703424; M. Beneke and V. A. Smirnov, Nucl. Phys. B 522, 321 (1998) arXiv:hep-ph/9711391.

97. K. G. Chetyrkin, J. H. Kuhn and M. Steinhauser, Nucl. Phys. B 482, 213 (1996) arXiv:hep-ph/9606230. R. Harlander, T. Seidensticker and M. Steinhauser, Phys. Lett. B 426, 125 (1998) arXiv:hep$\mathrm{ph} / 9712228$. A. H. Hoang and T. Teubner, Phys. Rev. D 58, 114023 (1998) arXiv:hepph/9801397]. K. Melnikov and A. Yelkhovsky, Nucl. Phys. B 528, 59 (1998) arXiv:hepph/9802379 . J. H. Kuhn, A. A. Penin and V. A. Smirnov, Eur. Phys. J. C 17, 97 
(2000) arXiv:hep-ph/9912503. M. Beneke, A. Signer and V. A. Smirnov, Phys. Lett. B 454, 137 (1999) arXiv:hep-ph/9903260. I. Blokland, A. Czarnecki and K. Melnikov, Phys. Rev. D 65, 073015 (2002) arXiv:hepph/0112267]. T. Becher and K. Melnikov, arXiv:hep-ph/0207201.

98. J. Kühn, in these proceedings.

99. A. Ghinculov and Y. P. Yao, Phys. Rev. D 63, 054510 (2001) arXiv:hep-ph/0006314.

100.G. Passarino and S. Uccirati, Nucl. Phys. B 629, 97 (2002) arXiv:hep-ph/0112004; G. Passarino, in these proceedings.

101.T. Binoth and G. Heinrich, Nucl. Phys. B 585, 741 (2000) arXiv:hep-ph/0004013.

102.D. E. Soper, Phys. Rev. D 62, 014009 (2000) arXiv:hep-ph/9910292; M. Kramer and D. E. Soper, Phys. Rev. D 66, 054017 (2002) arXiv:hep-ph/0204113; D. E. Soper, in these proceedings.

103R. K. Ellis, D. A. Ross and A. E. Terrano, Nucl. Phys. B 178, 421 (1981).

104.S. Catani, D. de Florian and M. Grazzini, JHEP 0105, 025 (2001) arXiv:hep$\mathrm{ph} / 0102227$.

105.M. Grazzini, in the proceedings arXiv:hep$\mathrm{ph} / 0209302$.

106.S. Dawson, Nucl. Phys. B 359, 283 (1991); A. Djouadi, M. Spira and P. M. Zerwas, Phys. Lett. B 264, 440 (1991).

107.M. A. Shifman, A. I. Vainshtein, M. B. Voloshin and V. I. Zakharov, Sov. J. Nucl. Phys. 30, 711 (1979) [Yad. Fiz. 30, 1368 (1979)].

108.M. Spira, Fortsch. Phys. 46, 203 (1998) arXiv:hep-ph/9705337.

109R. V. Harlander, Phys. Lett. B 492, 74 (2000) arXiv:hep-ph/0007289.

110R.E. Cutkosky, J. Math. Phys. 1, :429 (1960).

111.C. Anastasiou, L. Dixon and K. Melnikov, arXiv:hep-ph/0211141.

112.A. De Roeck, V. A. Khoze, A. D. Martin, R. Orava and M. G. Ryskin, Eur. Phys. J. C 25, 391 (2002) arXiv:hep-ph/0207042.

113.CMS collaboration, "CMS: The electromagnetic calorimeter, technical design report," report CERN/LHCC 97-33, CMS-TDR-4; ATLAS collaboration, "ATLAS detector and physics performance, technical design report," vol. 2, report CERN/LHCC 99-15, ATLAS-TDR-15.

114.V. Tisserand, "The Higgs to two photon decay in the ATLAS detector," talk given at the VI International Conference on Calorimetry in High-Energy Physics, Frascati (Italy), June, 1996, LAL 96-92; Ph.D. thesis, LAL 97-01, February, 1997.

115.M. Wielers, "Isolation of photons," report ATL-PHYS-2002-004.

116.E.L. Berger, E. Braaten and R.D. Field, Nucl. Phys. B 239, 52 (1984); P. Aurenche, A. Douiri, R. Baier, M. Fontannaz and D. Schiff, Z. Phys. C 29, 459 (1985); B. Bailey, J.F. Owens and J. Ohnemus, Phys. Rev. D 46, 2018 (1992); B. Bailey and J.F. Owens, Phys. Rev. D 47, 2735 (1993); B. Bailey and D. Graudenz, Phys. Rev. D 49, 1486 (1994) arXiv:hep-ph/9307368; C. Balazs, E.L. Berger, S. Mrenna and C.-P. Yuan, Phys. Rev. D 57, 6934 (1998) arXiv:hepph/9712471; C. Balazs and C.-P. Yuan, Phys. Rev. D 59, 114007 (1999) [Erratumibid. D 63, 059902 (1999) arXiv:hepph/9810319 ; T. Binoth, J.P. Guillet, E. Pilon and M. Werlen, Phys. Rev. D 63, 114016 (2001) arXiv:hep-ph/0012191; T. Binoth, arXiv:hep-ph/0005194.

117.T. Binoth, J.P. Guillet, E. Pilon and M. Werlen, Eur. Phys. J. C 16, 311 (2000) arXiv:hep-ph/9911340.

118.R.K. Ellis, I. Hinchliffe, M. Soldate and J.J. van der Bij, Nucl. Phys. B 297, 221 (1988).

119L. Ametller, E. Gava, N. Paver and D. Treleani, Phys. Rev. D 32, 1699 (1985); D.A. Dicus and S.S.D. Willenbrock, Phys. Rev. D 37, 1801 (1988).

120.D. de Florian and Z. Kunszt, Phys. Lett. B 460, 184 (1999) arXiv:hep-ph/9905283; C. Balazs, P. Nadolsky, C. Schmidt and C.P. Yuan, Phys. Lett. B 489, 157 (2000) arXiv:hep-ph/9905551.

121.T. Binoth, J.P. Guillet, E. Pilon and M. Werlen, arXiv:hep-ph/0203064. 